\title{
Effect of frequency, timbre, experience, and feedback on musical tuning skills
}

\author{
JOHN R. PLATT and RONALD J. RACINE \\ McMaster University, Hamilton, Ontario, Canada
}

\begin{abstract}
Subjects with tuning experience were superior to nontuners in a pitch-matching task at all but the lowest of five frequencies tested. Performance was better for adjustment of simple (sine-wave) tones to match simple tones than for adjustment of complex (string-timbre) tones to match simple tones. A comparison of constant errors showed that both tuners and nontuners heard complex tones as sharp relative to simple tones. Additional testing with 2 of the subjects indicated that the first overtone of the complex tone was sufficient to produce this effect. Repetition of the pitch-matching task over 6 days produced some improvement in performance. A second experiment showed that auditory and visual feedback improved tuning accuracy for complex comparison tones about 4.5 cents more than did practice without feedback, but no effect was observed for simple comparison tones. This experiment also indicated that musical experience, rather than tuning experience per se, was responsible for the effects of prior experience, and that tuning was more accurate when the standard was a complex tone and when the standard and comparison tones had the same timbre. The relevance of these data to musical tuning skills and to theories of complex pitch perception is discussed.
\end{abstract}

Although there has been a considerable amount of research on musical skills in the 20th century, much of this work has concentrated on highly skilled populations and has utilized the relatively complex materials of classical music. Much less attention has been devoted to the acquisition of very rudimentary musical skills. One such skill is the musician's ability to put his instrument in tune with a pitch standard. As indicated by Carlsen (1981), the extensive literature on pitch discrimination using simple tones does not provide information about the possible effects on perception of in-tuneness produced by the complex wave forms and amplitude envelopes of musical instruments. Sergeant (1973) has also argued that discrimination of simple tones does not provide an adequate test of musical tuning skills, and has suggested that a more realistic task would involve pitch discrimination between tones of different timbres such as a complex tone compared with a simple tone. The only modern study directly comparing frequency discrimination of musicians and nonmusicians (Spiegel \& Watson, 1984) found musicians to be superior with both simple and complex tones, but did not examine discrimination between tones of different timbre.

Many anecdotal observations suggest that timbre differences provide a major source of difficulty in the acquisition of tuning skills. For example, students learning to tune a stringed instrument often find it more difficult to

This research was supported by Grants A8269 and A8629 from the Natural Sciences and Engineering Research Council of Canada. The author's mailing aduress is: Department of Psychology, McMaster University, 1280 Main Street West. Hamilton, Ont. . Canada L8S 4K1. tune a string to a pitch standard, such as a tuning fork or pitch pipe, than to another string. Even when tuning one string to another on an instrument with wound and unwound strings, the greatest difficulty is usually found at the point of transition between the two types of strings.

In addition to such anecdotal evidence, several studies have reported systematic differences between the perceived pitch of simple and complex tones with the same fundamental frequency. Bannister (1934) says that the pitch of a complex tone is usually slightly higher than that of a simple tone with the same fundamental frequency, and that this difference is greater the more pronounced the overtones of the complex tone. Lichte (1941) found that subjects set a simple tone slightly sharp when adjusting it to match any of a variety of complex tones with fundamental frequencies of 360 or $540 \mathrm{~Hz}$, but not for tones of $180 \mathrm{~Hz}$. Using a very different task, Greer (1970) found that brass players tended to be flat when attempting to play in tune with a simple tone, but not when playing with an organ, a piano, or a recording of their own instrument. Both of these results are in agreement with Bannister's claim that the pitch of a complex tone is slightly higher than that of a simple tone of the same fundamental frequency. However, these results appear to conflict with several theories of pitch perception for complex tones (Goldstein, 1973; Terhardt, 1970; Wightman, 1973), which predict that a harmonic complex tone will be heard as slightly lower in pitch than a simple tone of the same fundamental frequency. Furthermore, this prediction has received support in at least one experiment (Terhardt, 1971).

Another point of some controversy concerning musical tuning skills has been their degree of trainability. 
Seashore (1938) believed that pitch discrimination was an inherited ability, not susceptible to improvement through training, and used this ability as a principle component of his musical aptitude tests. However, Wyatt (1945) showed that performance on several standardized tests of pitch discrimination, including Seashore's, was substantially improved by extended intonation and pitchdiscrimination training with feedback. Unfortunately, Wyatt's pitch-discrimination tests used simple tones or close approximations to simple tones, and in no case involved discrimination of pitch between tones of differing timbre. Also, Wyatt made no attempt to isolate the relative contribution of her various training tasks, or the importance of feedback to the obtained outcomes. A better controlled study by Campbell and Small (1963) found little, if any, effect of feedback on frequency discrimination, but they investigated only simple tones.

The first experiment in the present paper compared tuning of simple and complex tones with simple-tone standards over a wide range of standard frequencies, in populations with and without experience in the tuning of stringed instruments. The complex tones employed were synthesized to have string-like timbres. Standard and comparison tones were presented successively to avoid specialized tuning techniques based on beats or difference tones, and the method of adjustments was employed as a reasonable approximation to the skill of interest. The major purpose of this experiment was to determine the effects of timbre differences, experience, and frequency on tuning accuracy, with special attention to any systematic differences between the perceived pitch of simple and complex tones. The second experiment compared the effects of practice with and without feedback on tuning accuracy in populations with various kinds of musical experience.

\section{EXPERIMENT 1}

\section{Method}

Subjects. The 12 subjects ranged in age from 22 to 40 years and served voluntarily without monetary compensation. No audiometric screening was performed; however, subjects were asked to report any known or suspected hearing losses, and no positive responses were obtained. Four males and 1 female had had 4 or more years (mean $=7.5$ years) of experience with tuning one or more stringed instruments. Four males and 3 females had had no such experience, although 3 of them had had 2 to 10 years' experience playing piano. These two groups will be referred to as "Tuners" and "Nontuners," respectively. Classification of the three piano players as Nontuners might be questioned. Although these subjects had not been directly involved in tuning procedures, they would be expected to be experienced in judging the integrity of their instrument. However, there is good reason to believe that tuning involves more than a judgment of integrity. For example, it is much easier to detect a pitch difference than to identify the direction of that difference (Ward, 1970). Although the former should be sufficient for a judgment of integrity, both are clearly required to make an appropriate tuning adjustment. This issue will be examined more closely in Experiment 2.

Apparatus. The subjects were seated comfortably in a soundattenuating room. Experimental control and data acquisition were performed by an Apple II-Plus computer. Auditory stimuli were generated by a Mountain Computer Music System consisting of 16 digital oscillators. Each oscillator was an 8-bit digital-to-analog converter controlled by a 256-byte waveform table at a sample rate of $31.25 \mathrm{kHz}$ with 1-byte amplitude and 2-byte frequency specifications. The outputs of all oscillators were combined by a summing amplifier, low-pass filtered for smoothing, passed through a buffer amplifier to a Yamaha CA-410 audio amplifier, and presented to both ears through Realistic PRO-1 earphones. The cutoff frequency of the low-pass filter was approximately $15 \mathrm{kHz}$, and the earphones had a relatively flat frequency response to $15 \mathrm{kHz}$. The highest frequency component in any stimulus used in this experiment was $9.6 \mathrm{kHz}$.

Instructions and prompts were presented on a Zenith ZVM-121 monochrome video monitor located at eye level, directly in front of the subject. A hand-held unit, containing a one-turn potentiometer and a push button, was used by the subject to adjust the comparison frequency and to indicate a pitch match.

Stimuli. The simple tones in this experiment were sine waves with amplitude envelopes of 512-msec duration, including a 1-msec rise time and 32-msec fall time. Complex tones were bandlimited sawtooth waves produced by additive synthesis of the first eight partials in phase, the amplitude of each partial being weighted by the reciprocal of its partial number. This waveform is similar to that produced by a bowed string and is also a crude approximation to the average waveform for the dynamically more complex spectrum of a plucked string. As with the simple tones, the complex tones had amplitude envelopes of 512-msec duration with a 1-msec rise time. However, the complex tones decayed throughout their duration according to the double exponential

$$
A_{t}=64 e^{-t / 112}+40 e^{-t / 714} \text {, }
$$

where $t$ is time in milliseconds and $e$ is the base of the natural logarithms. This amplitude envelope is similar to one that could be produced by a plucked string.

The amplitude of the simple tones was adjusted to a relatively loud, but comfortable, listening level. This level was measured at the earphones, with the aid of an artificial ear, to be $72 \mathrm{~dB}$ SPL at each frequency employed. Equal loudness contours for simple tones of this intensity are nearly flat across the frequency range employed in this study (Fletcher \& Munson, 1933). It is thus reasonable to argue that the various simple tones used in this study were equated for loudness.

Controlling the loudness of the complex tones was more problematic, since scaling data were not available for the particular complex tones employed here. Both experimenters independently and repeatedly adjusted the level of the complex tones to match the loudness of the simple tones. These adjustments appeared to be very reliable and essentially identical for the two experimenters. The mean of these adjustments was used to set the level of the complex tones. The resulting tones, presented continuously at the maximum value of their amplitude envelopes, were $75 \mathrm{~dB}$ SPL at the earphones.

Procedure. Every trial consisted of an alternating sequence of a simple standard tone and either a simple or a complex comparison tone. The two tones were separated by a $512-\mathrm{msec}$ silent interval, and the pair was repeated after a $1-\mathrm{sec}$ silent interval. The subject was instructed to adjust the pitch of the comparison tone until it matched the standard, and then to push a button indicating the match and ending the trial. At the beginning of each trial, the subject was instructed to adjust the potentiometer until it was physically centered. This was accomplished by using a numerical display on the video screen with positive and negative numbers corresponding to magnitudes of clockwise and counterclockwise deviation, respectively. This position was then randomly assigned a comparison frequency $\pm 1 \%$ to $\pm 3 \%$ of the standard frequency so that the correct position would vary from trial to trial. The potentiometer could then be used to adjust that initial comparison frequency $\pm 6 \%$ with 1 $\mathrm{Hz}$ resolution.

The five standard frequencies employed were $115,205,370,670$, and $1200 \mathrm{~Hz}$. These frequencies were chosen to be spaced approximately logarithmically, to avoid notes of the equally tempered scale, 
and to include the pitch range of most stringed instruments except those with very low registers. Trials were always presented in an ascending sequence of standard frequencies with complex comparison tones, followed by a similar sequence with simple comparison tones. Six such sequences were presented in a session which lasted approximately $1 \mathrm{~h}$.

Each subject participated for six consecutive daily sessions for a total of 36 trials with each comparison tone at each standard frequency. Data recorded on each trial included the starting frequency of the comparison tone, the frequency at which the subject indicated a pitch match, and the number of standard-comparison pairs required to obtain the match.

Two of the Tuners were run for two additional sessions. During the first of these sessions, the complex tone was modified to have the same amplitude envelope as the simple tone-512-msec duration, including a $1-\mathrm{msec}$ rise time and a $32-\mathrm{msec}$ fall time-but all partials were still present. During the second session, the complex tone was further modified to eliminate all partials beyond the second. At this point, the complex and simple tones differed only in the presence versus absence of a first overtone with twice the frequency and half the amplitude of the fundamental. All other aspects of the procedure remained the same as in the main experiment.

\section{Results and Discussion}

The subjects listened to a mean of $13.7 \pm 0.6$ standardcomparison pairs ${ }^{1}$ before indicating a pitch match. This value is smaller than the 40 pairs reported as typical by Wier, Jesteadt, and Green (1976) for subjects adjusting the pitch of a simple tone to match a $1-\mathrm{kHz}$ simple-tone standard. The difference may reflect their use of a 10turn, instead of a 1-turn, potentiometer to adjust the comparison frequency. In any case, the difference would be of concern only if it produced a difference in estimates of accuracy. Data to be presented below suggest that it did not.

The number of standard-comparison pairs required for a match was not systematically affected by tuning experience or frequency of the standard tone. However, slightly more pairs were required to obtain a match when the comparison was a simple tone $(15.0 \pm 0.8)$ than when it was a complex tone $(12.4 \pm 0.5)$. This difference probably reflects the subjects' greater sensitivity to frequency differences with simple-tone pairs.

The results of primary interest were those reflecting the accuracy with which subjects matched comparison to standard frequencies. To facilitate evaluation of errors within a musical context, all comparison settings were first transformed from hertz to cents. One cent is one hundredth of a semitone, and thus bears a logarithmic relationship to frequency in hertz. In the present case, cents were computed from the ratio of comparison to standard frequency. Thus, positive values indicated sharpening of the comparison with respect to the standard and negative values indicated flattening. Three summary measures of errors were examined. Absolute error was computed as the mean comparison setting, without regard to sign. Although this is probably the simplest overall measure of in-tuneness, it could be inflated by either a constant tendency to err in a particular direction or by random variability in comparison settings. To separate these two sources of variance, two additional measures of accuracy were used.
Constant error was computed as the mean comparison setting, and relative error was computed as the standard deviation of the comparison settings.

Examination of the three error measures over the course of the experiment revealed relatively small practice effects except for some incidence of unusually large errors on the first few trials with complex comparison tones, particularly in Nontuners. As a typical example, Figure 1 plots mean absolute error against sessions for each of the $670-\mathrm{Hz}$ conditions in the experiment. As can be seen, Nontuners showed most of their improvement in the setting of complex tones from the first to the second session.

Because of the impracticality of graphically presenting a complete picture of practice effects, two statistical analyses were performed to evaluate them. An unweighted means analysis of variance was performed on absolute errors in which the variables were experience (Tuners vs. Nontuners), timbre (simple vs. complex), frequency, and sessions. The effect of sessions was significant $[F(5,50)=$ $11.54, \mathrm{p}<.001$ ], indicating a decrease in magnitude of error with practice. The interactions of sessions with experience $[F(5,50)=4.04, p=.004]$ and with timbre $[F(5,50)=2.58, p=.037]$ were also significant, indicating the larger practice effects for complex tones and for Nontuners. A similar analysis of variance was performed excluding the first session. This analysis still yielded a significant sessions effect $[F(4,40)=4.46, p=.004]$ but no significant interactions of sessions with other variables. On this basis, all subsequent analyses were based on mean performance over the last five sessions of the experiment, during which time each subject received 30 trials with each comparison stimulus at each frequency. It is also important to note that the practice effects obtained were relatively small. The overall mean absolute error decreased from 20 cents in the first session to 16 cents in the second. The remaining sessions of practice produced only a 2 -cent decrease to 14 cents in the sixth session.

Figure 2 plots means of each of the three error measures as a function of standard frequency, with simple versus complex comparison tones and tuning experience as parameters. In agreement with the hypothesis that timbre differences are a major source of difficulty in tuning, all three error measures yielded larger values for complex comparison tones than for simple ones. The notion that individuals who have successfully acquired tuning skills would have minimized this difficulty is supported by the smaller differences between simple versus complex comparison tones in Tuners, particularly in terms of relative error.

The absolute errors for Tuners and Nontuners were essentially identical at a standard frequency of $115 \mathrm{~Hz}$. However, as standard frequency increased, absolute errors remained relatively constant for Nontuners, but decreased for Tuners. In addition, absolute error functions for simple versus complex comparison tones were approximately parallel across the frequency dimension for both Tuners and Nontuners. 


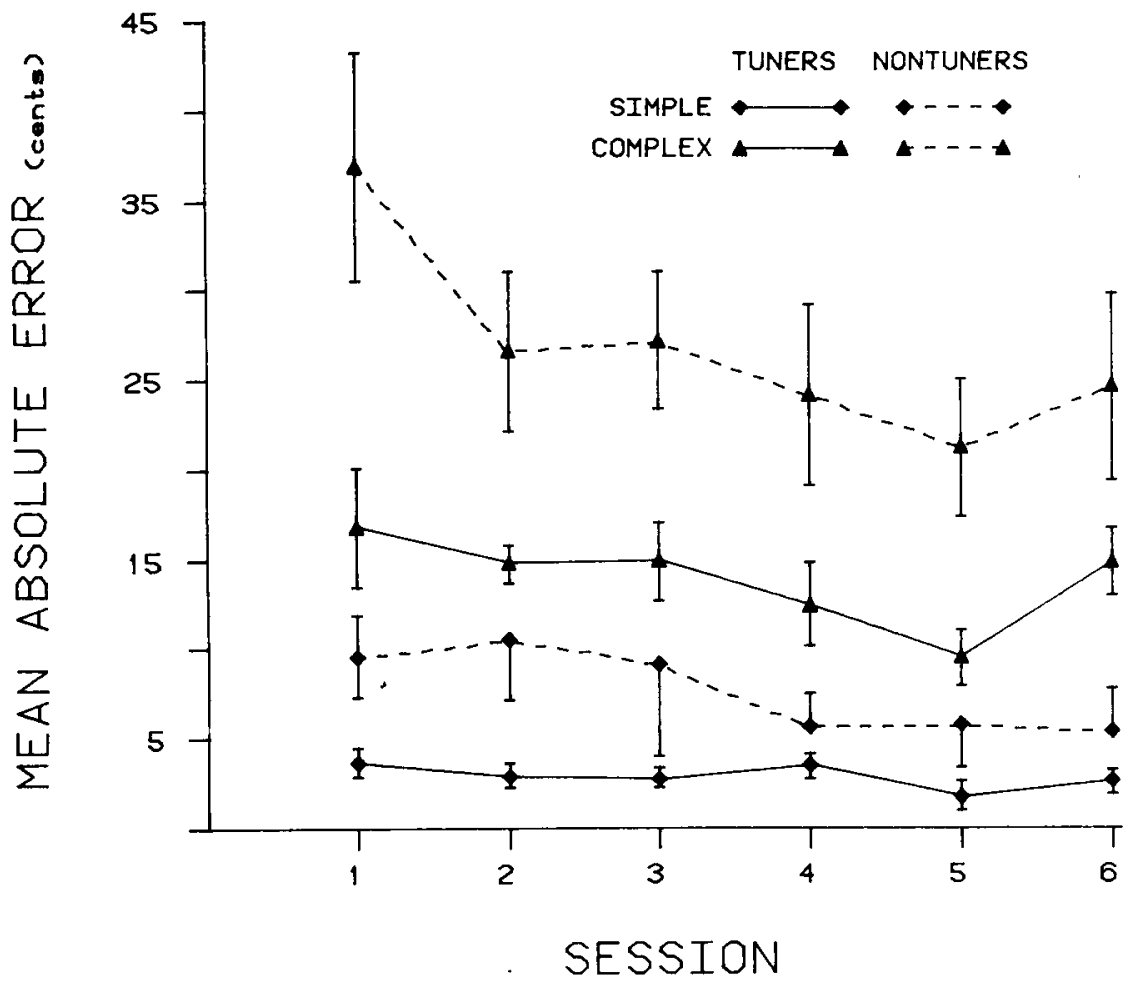

Figure 1. Mean absolute error in cents as a function of sessions for the $670-\mathrm{Hz}$ tones in Experiment 1.

Relative errors displayed the same general functional relationships as absolute errors. Differences in absolute errors between Tuners and Nontuners were almost entirely accounted for by differences in relative error. The relative error data can also be compared with those from simple-pitch-discrimination studies. For example, Wier et al. (1976) reported that highly practiced subjects yield a mean standard deviation of $1.1 \mathrm{~Hz}$ when adjusting a simple tone to match a $1-\mathrm{kHz}$ simple-tone standard. In the present study, Tuners adjusting simple tones yielded mean standard deviations of 1.0 and $1.7 \mathrm{~Hz}$ at 670 and $1200 \mathrm{~Hz}$, respectively. When these values are expressed relative to standard frequency to obtain Weber fractions, all three yield values of .001 .

Turning to the constant error measure in the middle panel of Figure 2, the performance of Tuners and Nontuners was surprisingly similar. Both groups showed negligible amounts of constant error when adjusting simple comparison tones. What little constant error there was tended to be positive and did not appear to be systematically related to standard frequency. There was a tendency to set complex comparison tones flat, which presumably reflects hearing them sharp. This tendency was most pronounced at intermediate frequencies. The 9- to 13-cent maximum average amount of flattening would be of questionable musical significance; however, the result may be surprising in a psychoacoustical framework.
Central pitch processor theories (Goldstein, 1973; Terhardt, 1970; Wightman, 1973) predict that a harmonic complex tone will be heard as slightly lower in pitch than a simple tone with a frequency equal to the fundamental of the complex tone. Several studies have supported this prediction, including one by Terhardt (1971) which used procedures and stimuli similar to those of the present experiment. When subjects adjusted the frequency of a simple tone to match the pitch of a complex tone, the simple tone was set lower than the fundamental frequency of the complex tone. The magnitude of this effect decreased from about 50 cents at $50 \mathrm{~Hz}$ to 0 cents above $500 \mathrm{~Hz}$. This result appears contradictory to the present result, and the only obvious difference between the studies appears to be which tone was the standard and which the comparison.

A study by Lichte (1941) suggests that the discrepancy between the present result and Terhardt's (1971) is not attributable to which tone served as standard and which served as comparison. Lichte had subjects adjust a simple tone to match the pitch of a variety of complex tones with up to 16 harmonic components. At a fundamental frequency of $180 \mathrm{~Hz}$, there were no systematic constant errors. However, at 360 and $540 \mathrm{~Hz}$, there was a marked tendency to set the simple-tone comparison to a frequency higher than the fundamental of the complex-tone standard. Thus, just as in the present study, subjects appeared to hear complex tones with fundamental frequencies of several hundred hertz as higher pitched than correspond- 


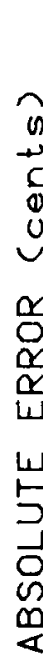

TUNERS

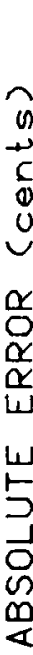

40

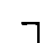

7

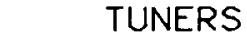

$\hat{\imath}$

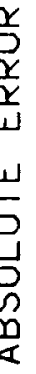

$\frac{n}{0}$

$\frac{\alpha}{0}$

占

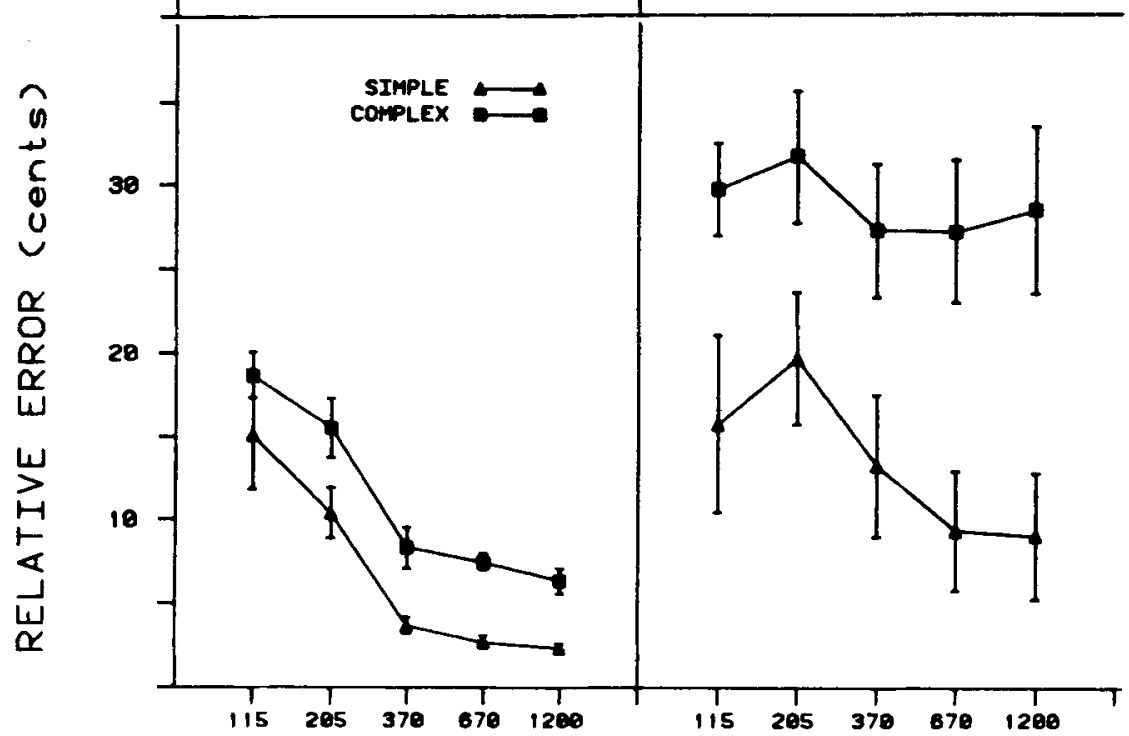

FREOUENCY OF STANDARD (HZ)
NONTUNERS
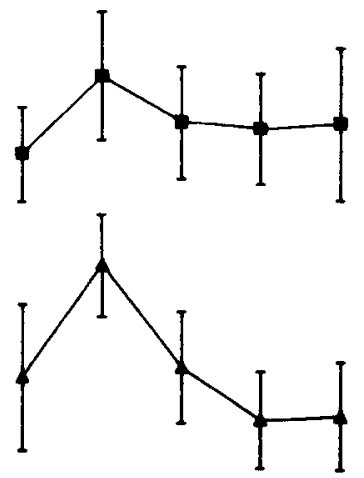

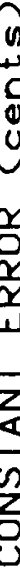

12

o

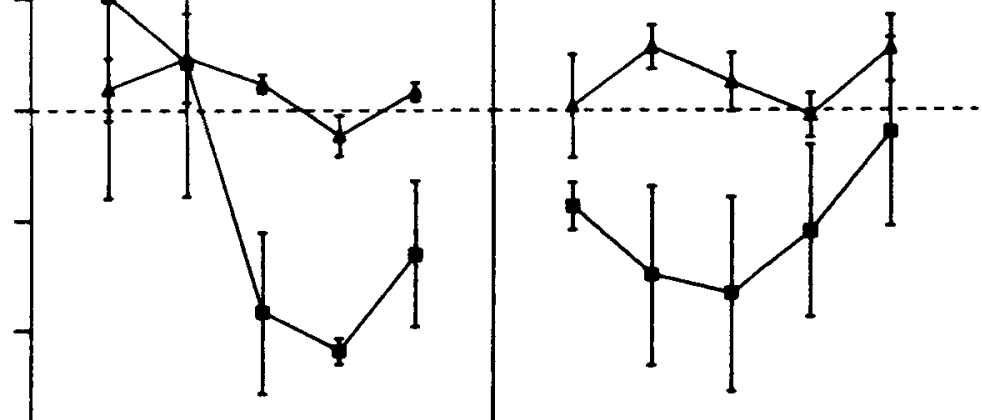

10

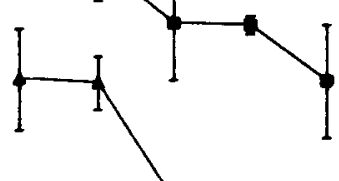


ing simple tones at the same fundamental frequency. A similar finding with a very different task was reported by Greer (1970). He examined brass players' ability to play in tune with a simple-tone generator, an organ, a piano, or a recording of their own instrument. There were no systematic constant errors except in the case of the simpletone standard, to which the subjects played an average of about 20 cents flat.

The two additional sessions run for 2 of the Tuners represented an attempt to isolate the aspects of the complex tone that were responsible for its being heard as higher pitched than a simple tone at the same fundamental frequency. Although individual differences in this tendency were quite large for Nontuners (a range of approximately 2 to 30 cents at maximum), Tuners showed much less between-subject variability (a range of approximately 11 to 22 cents at maximum). Thus, the 2 Tuners, who were selected for further study on the basis of their availability, were quite typical of the entire sample of Tuners with respect to the magnitude of this flattening effect.

The general strategy in these additional sessions was to successively make the complex tone more like the simple tone while looking for a reduction in the tendency to hear the former as higher pitched. Figure 3 plots constant errors as a function of standard frequency for these 2 subjects. Separate functions are plotted for simple and com- plex tones and for each of the two additional sessions. The constant errors for each of these subjects over the last five sessions of the main experiment are also presented for comparison. It should be noted that J.P.'s tendency to sharpen at $205 \mathrm{~Hz}$ was not observed in any other subject in the experiment. Subsequent audiometric testing of this subject yielded a perfectly "normal" audiogram for a 40-year-old subject.

Despite the relatively small number of observations involved, it is clear from Figure 3 that neither equating the amplitude envelopes of the simple and complex tones (Session 1) nor also eliminating all harmonic content beyond the first overtone (Session 2) had any appreciable effect on the pattern of constant errors. That is to say that the mere presence of a single overtone was sufficient to raise the pitch above that associated with the fundamental frequency. This result is in general agreement with the results of Greer (1970) and Lichte (1941), in that both found the higher pitch of complex tones to hold across a variety of such tones differing in amplitude and spectral envelopes.

\section{EXPERIMENT 2}

Experiment 1 showed large differences between the tuning accuracy of Tuners and Nontuners, and some improvement with practice for both groups. No trial-by-trial

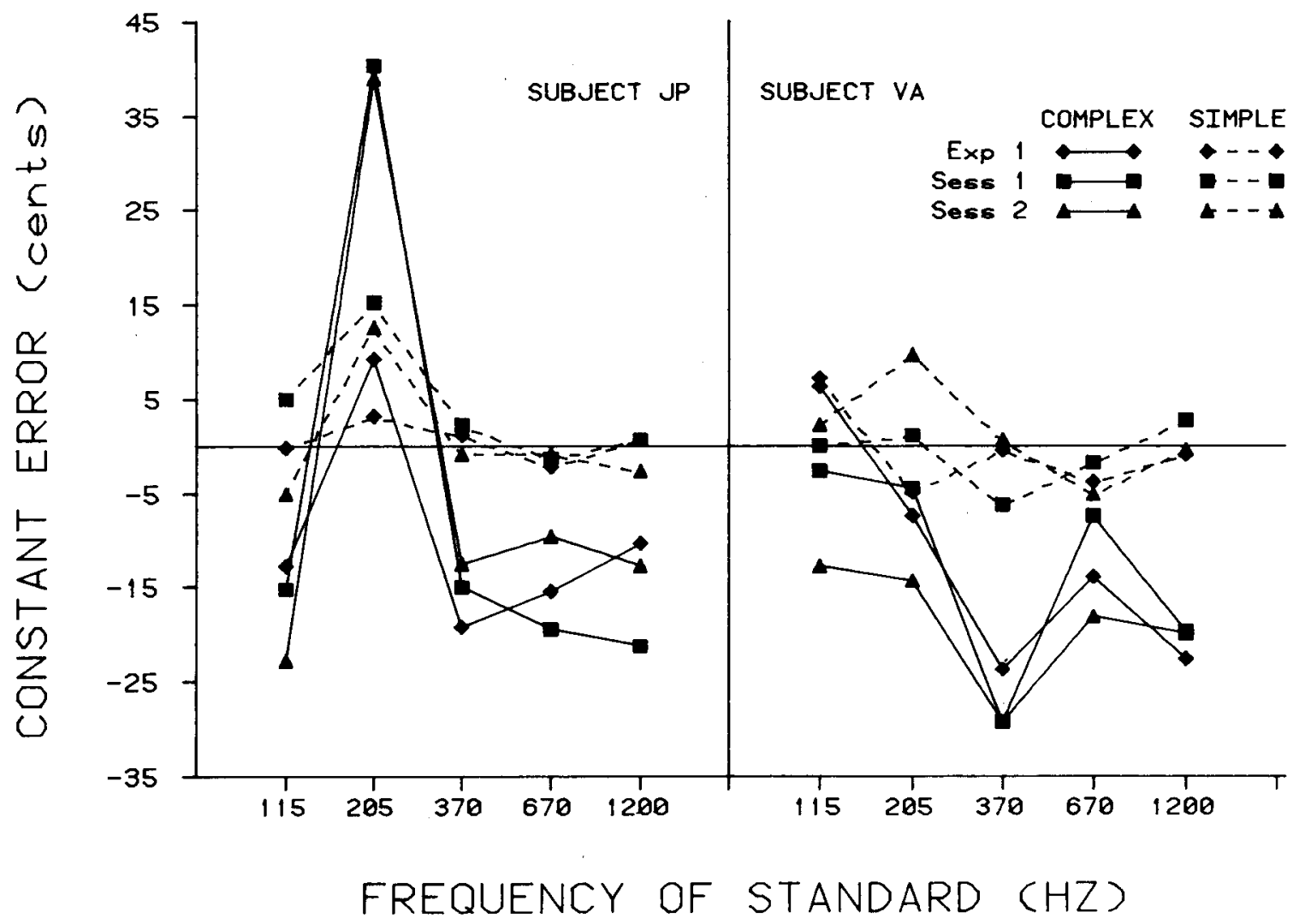

Figure 3. Mean constant error in cents as a function of fundamental frequency for the two subjects tested on complex tones with simplified amplitude envelopes and harmonic content. Note that spacing on the abscissa is logarithmic. 
feedback or other knowledge of results was provided in that experiment. As indicated in the introduction, the wellcontrolled study by Campbell and Small (1963) found no effect of feedback on pitch discrimination. However, Campbell and Small did not investigate complex tones or standard-comparison pairs involving differing timbres. The present experiment examined the effects of feedback training on the tuning of stimuli like those used in Experiment 1 .

Experiment 2 also investigated two additional issues raised by Experiment 1 . The lower tuning accuracy found for complex tones was attributed to timbre differences between standard and comparison stimuli; however, it could have been an effect of complex tones per se. These alternatives were examined in the present study by testing all combinations of simple or complex standards and simple or complex comparisons, both before and after training. Finally, there was some ambiguity in Experiment 1 as to the classification of musically experienced subjects who were not experienced at tuning an instrument. That is, it was impossible to determine whether the beneficial effects of experience were correlated with tuning experience, or with musical experience in general. The present experiment addressed this issue by investigating groups of musically experienced subjects with and without tuning experience, as well as a group of musically inexperienced subjects.

\section{Method}

Subjects. The subjects were 32 students in an undergraduate perception course who participated as part of their laboratory work for the course. Fourteen of these subjects had had less than 1 year of training on any musical instrument, and 10 of those had had no experience. Nine subjects had had 4 or more years (mean $=9$ years) experience on one or more instruments that did not involve tuning. Nine additional subjects had had 4 or more years (mean $=11$ years) experience on one or more instruments, at least one of which they regularly tuned. These three groups will be referred to as "Inexperienced," "Nontuners," and "Tuners," respectively. It is important not to confuse the present Nontuner group with that in Experiment 1 , which, in the present terminology, was a mixture of Inexperienced subjects and Nontuners.

All of the subjects were screened using a Békésy-type audiometer (Bruel and Kjaer, Model 1800). All except one of the subjects showed "normal" hearing in that they tested at $20 \mathrm{~dB}$ HL or better in both ears at all test frequencies. One Inexperienced subject tested at $40 \mathrm{~dB} \mathrm{HL}$ at $3000 \mathrm{~Hz}$ and above in the left ear. It was thought that this mild hearing loss in one ear should provide no problem since experimental stimuli were to be presented to both ears. Subsequent examination of this subject's experimental results revealed no noticeable departures from those for other subjects under the same conditions.

Apparatus. The apparatus was essentially the same as that used in Experiment 1, with the exception of the substitution of Realistic Nova-PRO earphones for the PRO-1 earphones. This substitution was made necessary by the use of multiple experimental stations in the present experiment and the unavailability of additional PRO-1 earphones. The Nova-PRO earphones produced some attenuation of the highest harmonics of the complex tones as compared with the PRO-1 earphones. This was not thought to be problematic, since the results of Experiment 1 had suggested that the fundamental and first overtone of the complex tone were sufficient to produce the differences observed between the simple and complex tones.
Stimuli. The stimuli were the same as those used in Experiment 1, except that their duration was decreased from 512 to $500 \mathrm{msec}$ and the fundamental frequency of the standard was fixed at $670 \mathrm{~Hz}$. This frequency was chosen because it had produced near maximum negative constant errors when complex comparison tones were tuned to simple-tone standards in Experiment 1.

Procedure. The general procedure was similar to that for Experiment 1 . In the first session, all subjects were treated identically. On each of 48 trials, they were required to adjust the frequency of the comparison tone to match the pitch of the standard. There were 12 trials with each of the four possible combinations of simple or complex standard and simple or complex comparison. No feedback or knowledge of results was provided.

Each experience group was then randomly and equally divided into a feedback and a no-feedback group. In experience groups with an odd number of subjects, the additional subject was assigned to the feedback group. One week later, all subjects were run for five additional daily sessions of $\mathbf{4 8}$ trials each. The first four of these sessions used only simple-tone standards, 24 trials with a simple-tone comparison and 24 with a complex-tone comparison. Subjects in the feedback condition received trial-by-trial auditory and visual feedback. One second after the subject indicated a pitch match, a vertical bar, representing the frequency of the standard, was displayed on the video screen and the standard tone was presented for $500 \mathrm{msec}$. After $500 \mathrm{msec}$ of silence, a second vertical bar representing the subject's setting of the comparison to a maximum of $\pm 20 \mathrm{~Hz}$ of the standard frequency was added to the video display and the comparison was presented for $500 \mathrm{msec}$ at the frequency set by the subject. Immediately after this, the comparison bar was redrawn to be the same height as the standard bar and the comparison tone was presented for $500 \mathrm{msec}$ at the same frequency as the standard.

The sixth and last session of the experiment was identical to the first session. All subjects were treated identically. All four possible combinations of simple or complex standard and simple or complex comparison were employed, and no feedback or knowledge of results was provided. All other details of the procedure were the same as for Experiment 1.

\section{Results and Discussion}

Analysis of results was concentrated on absolute errors as an overall measure of tuning accuracy. Relative and constant errors will be mentioned only when they reveal pertinent additional information. The effects of practice and of feedback were ascertained by comparing performance on the first and last sessions of the experiment. Although no subject received feedback during these two sessions, they were chosen for analysis because the interest was in "associative" effects of feedback which would persist after feedback was discontinued. It was specifically desired to disregard "nonassociative" effects of feedback, such as any possible tendency to overcorrect on trial $n$ on the basis of the feedback on trial $n-1$.

Figure 4 shows the mean absolute errors on Sessions 1 and 6 for all the conditions in the experiment. Because of the complexity of the experimental design and the large number of between-subject comparisons involved, an analysis of variance was performed on the means displayed in Figure 4. This was an unweighted means analysis in which the variables were experience (Inexperienced vs. Nontuners vs. Tuners), feedback (feedback vs. no feedback), standard (simple vs. complex), comparison (simple vs. complex), and sessions (1 vs. 6). Presentation of results will be organized around the statistically 


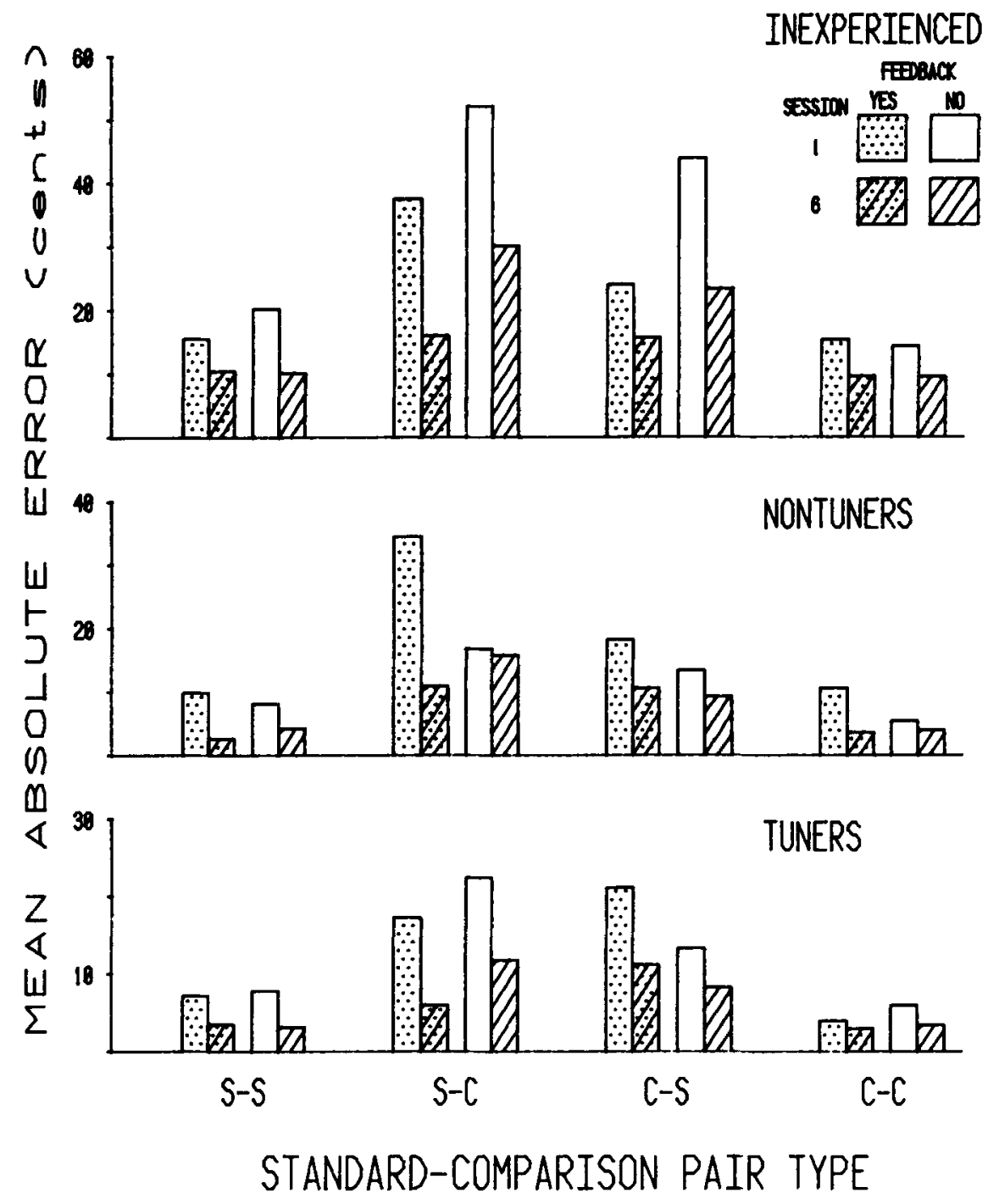

Figure 4. Mean absolute error in cents for the first and last sessions of each condition in Experiment 2.

significant effects revealed by this analysis; any effect not mentioned was not statistically significant.

The analysis yielded a significant effect of experience $[F(2,26)=4.40, p=.002]$. Planned comparison showed this effect to result from Inexperienced subjects' having larger absolute errors than either Nontuners or Tuners $[F(1,26)=9.88, p<.005]$, who did not differ from each other $[F(1,26)<1.0]$. This result indicates that the present benefits of experience are attributable to musical experience in general, rather than to tuning experience per se, and is in general agreement with Spiegel and Watson's (1984) finding that frequency discrimination, using the method of constant stimuli, was better for musicians than for nonmusicians, but did not differ as a function of whether a musician was experienced at tuning an instrument. These results, taken together, suggest that classification of the three piano players with the musically inexperienced subjects in Experiment 1 was inappropriate, and that the large effects of experience obtained there may have been underestimated.

The analysis also yielded a significant effect of sessions $[F(1,26)=27.27, p<.001]$, which reflects the improvement in tuning accuracy with practice in all conditions of the experiment. The overall mean absolute error decreased from 18.4 cents in Session 1 to 9.9 cents in Session 6. In spite of a number of differences in conditions, the magnitude of this effect is similar to that obtained in Experiment 1, in which the mean absolute error decreased from 20 to 14 cents over six sessions.

There were several statistically significant effects involving the nature of the standard and comparison stimuli. Interpretation of these effects is aided by the upper frame of Figure 5, which plots mean absolute error in Sessions 1 and 6 for each combination of simple or complex stan- 


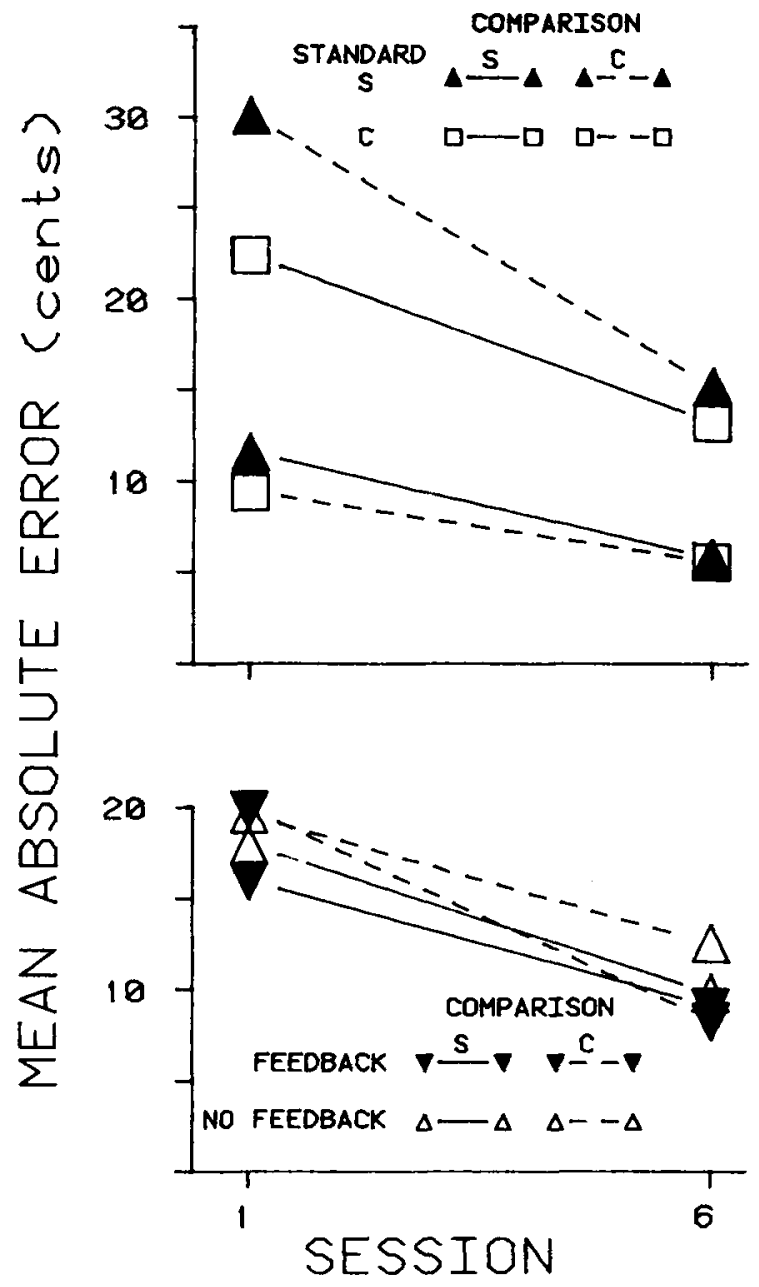

Figure 5. Mean absolute error in cents for the first and last sessions of Experiment 2. The upper frame shows means for all combinations of standard and comparison stimuli. The lower frame shows means for all combinations of feedback and comparison stimulus.

dard and simple or complex comparison. Tuning accuracy was higher with complex than with simple standard tones $[F(1,26)=12.02, p=.002]$, but this difference decreased from 5.0 to 1.1 cents with practice $[F(1,26)$ $=6.52, p=.016]$. This result is in general agreement with the frequent finding that pitch discrimination is better for complex than for simple tones (Henning \& Grosberg, 1968; Sergeant, 1973; Spiegel \& Watson, 1984; Zeitlin, 1964). These previous studies only investigated same-timbre standard-comparison pairs. The use of a factorial design in the present study allows the additional conclusion that the superiority of pitch discrimination with complex tones is entirely attributable to the nature of the standard tone. A complex tone stimulates a wider pattern of neural activity beginning at the level of the cochlea. The superiority of pitch discrimination with complex tones may reflect this additional, even if largely redundant, information's functioning to facilitate accurate memory of the standard.
In addition to the simple effect of the nature of the standard stimulus, there was an even larger standardcomparison interaction $[\mathrm{F}(1,26)=30.68, \mathrm{p}<.001]$, which decreased somewhat with practice $[F(1,26)=8.67$, $p=.006]$. As can be seen in Figure 5, this interaction reflects the greater tuning accuracy obtained when standard and comparison stimuli had the same timbre. This replicates the finding of greater tuning accuracy for simple-simple than for simple-complex tone pairs in Experiment 1 , and makes clear that it was an effect of timbre similarity rather than of complex tones per se. The larger absolute errors produced when standard and comparison stimuli differed in timbre were based on both larger relative errors and larger constant errors. In particular, the tendency found in Experiment 1 for complex tones to be heard as higher pitched than simple tones with the same fundamental frequency was replicated. In the present experiment, the complex tone was set an average of 17.8 cents flat when it was tuned to a simple-tone standard in Session 1. Conversely, the simple tone was set an average of 10.2 cents sharp when it was tuned to a complex-tone standard. These constant errors were reduced, but not eliminated, by practice. Average constant errors were consistently 1 cent or less, however, when standard and comparison stimuli had the same timbre.

The effects of feedback were not large in the present experiment. The only statistically significant effect of feedback was its interaction with comparison and session $[F(1,26)=4.80, p=.035]$. This interaction is plotted in the lower frame of Figure 5. Practice with feedback produced about 4.5 cents more improvement than did practice without feedback when the comparison tone was complex, but not when it was simple. Although this effect is apparent only for Nontuners in Figure 4, its interaction with experience only approached statistical significance $[F(2,26)=2.51, p=.10]$. The present results with respect to feedback agree with those of Campbell and Small (1963) in showing no effect for simple tones. Small effects are demonstrable for complex comparison tones, however, at least for Nontuners. These are, of course, exactly the conditions of greatest relevance to the training of musical tuning skills.

\section{GENERAL DISCUSSION}

The present experiments carry implications both for musical tuning skills and for psychoacoustical theories of pitch perception. On the musical side, these results offer support to those who have questioned the relevance of simple-tone discrimination to tuning skills (Carlsen, 1981; Sergeant, 1973). Many of the effects found in the present experiments required the inclusion of complex tones and of standard-comparison tone pairs involving timbre differences. Tuning was consistently less accurate and displayed systematic constant errors when standard and comparison stimuli differed in timbre. The results of the addendum to Experiment 1, together with results discussed 
earlier (Greer, 1970; Lichte, 1941), suggest that the constant errors resulting from timbre differences are primarily attributable to the presence versus absence of overtones, rather than to more subtle timbre differences. Experiment 2 indicated that the greater accuracy of tuning typically found with complex tones is actually an effect of complex-tone standards. These two results taken together lead to the recommendation that musical tuning will be most accurate if one avoids the use of pitch standards that are simple, or near-simple, tones, such as sine-wave oscillators and tuning forks.

Turning to the training of tuning skills, the effects of feedback in Experiment 2 were not very dramatic. In agreement with earlier work (Campbell \& Small, 1963), feedback had no effect on the tuning of simple comparison tones. Small improvements in tuning accuracy were produced by feedback in the musically more relevant case of complex comparison tones-mainly for musically experienced subjects without prior tuning experience. It is likely that further research that systematically varies the nature of feedback employed would lead to larger benefits from feedback training. For example, the visual feedback used in Experiment 2 was quantitatively limited to $\pm 20 \mathrm{~Hz}$. It was often observed that extremely inaccurate subjects would simply alternate between larger errors in opposite directions, effectively receiving only directional information about their errors and expressing considerable frustration and discouragement. This problem could easily be eliminated by the use of a feedback display with a wider range of values, or even a range of values and resolution tailored to the subject's current level of performance. In any case, the finding, both here and in Spiegel and Watson (1984), that the performance of musically experienced subjects on tuning or frequency discrimination tasks is independent of their tuning experience suggests that training of tuning skills is not a long-term problem in musical training. All that really seems to be needed is an efficient way to bring the skill to a suitable level to begin musical training.

With respect to the more general issue of the trainability of tuning skills, the beneficial effects found for musical experience, practice, and feedback are consistent with the view that tuning skills are at least to some extent trainable. On the other hand, in the absence of longitudinal or genetic data, it is impossible to estimate the possible contribution of nativistic components to this skill. After six sessions of experience with the task, musically inexperienced subjects were still inferior to experienced ones. This suggests that either the skill requires an extended period of training, or that it has nativistic components that are correlated with the likelihood of pursuing musical training. Even the improvements found with practice in the present task might have had more to do with learning about situational specifics than with improvements in pitch discrimination (Seashore, 1938). The fact that the bulk of these practice effects occurred very rapidly and fell far short of accounting for the total difference between ex- perienced and inexperienced subjects would be consistent with this interpretation.

The present results also offer several implications for psychoacoustic theories of pitch perception. As indicated previously, it has often been reported that frequency discrimination is better for complex than for simple tones. The greater information content of complex tones, the wider patterns of neural activity they stimulate, and the involvement of centrally inferred virtual pitches (Terhardt, Stoll, \& Seewann, 1982) offer many possibilities for interpreting this superiority. The present finding that this superiority is entirely due to the standard stimulus serves to limit these possibilities to functions that are involved in processing the standard, but not the comparison, stimulus. Perhaps the most obvious of such functions would be the necessary involvement of pitch memory in mediating the temporal gap between standard and comparison stimuli. The greater redundancy of pitch information in harmonic complex tones may serve to facilitate this memory function.

Another psychoacoustically interesting aspect of the present results was the tendency for complex tones to be perceived as slightly higher pitched than simple tones at the same fundamental frequency - an effect that was maximal at frequencies of several hundred hertz. This effect is in agreement with other studies of tuning (Lichte, 1941) and intonation (Greer, 1970), but conflicts with results of some psychoacoustical studies (e.g., Terhardt, 1971) directed to testing central pitch processor theories (Goldstein, 1973; Terhardt, 1971; Wightman, 1973). The Terhardt study found a tendency for complex tones to be perceived as slightly lower in pitch than simple tones at the same fundamental frequency - an effect that was maximal at the lowest frequency tested and nonexistent above $500 \mathrm{~Hz}$.

Terhardt et al.'s (1982) extension of the Terhardt (1971) model for pitch extraction from complex tonal signals provides a possible framework for considering these conflicting results. The quantitative details of this model are complex, but the basic idea is that various spectral and virtual (centrally inferred) pitches are extracted from a complex tone and compete with each other. The model computes weights to represent the relative prominence of these various pitches. An important aspect of the model is that two perceptual modes compete, an analytic mode involving "hearing out" spectral pitches and a holistic mode, similar to that specified by the previously mentioned central pitch processor theories, which results in a dominant virtual pitch with timbre. In its present form, the weights computed by the model are designed to describe the relative prominence of pitches within the spectral and virtual groups, but not between the groups. The model is also silent as to exactly how these various weights would be resolved into a single comparison-stimulus setting when several pitch percepts are in competition.

Despite the present limitations of the Terhardt et al. model, it clearly predicts that a harmonic complex tone 
will be heard as slightly lower pitched than a simple tone at the same fundamental frequency if the holistic perceptual mode predominates. This prediction is arrived at in essentially the same manner as in the earlier central pitch processor theories. The model could also deal with the present finding of higher pitches for complex tones if it were assumed that (1) the analytic perceptual mode predominated, (2) the fundamental and first overtone provided the dominant spectral pitches, and (3) the conflict between these two percepts was resolved into a single comparison-stimulus setting by tuning to the fundamental but accommodating the setting slightly in the direction of the first overtone. Some credence is given to this interpretation by the obtained pattern of constant errors over the frequency dimension. A primary determinant of spectral pitch weights in Terhardt et al.'s model is the frequency of the spectral component. This spectral dominance phenomenon increases to about $700 \mathrm{~Hz}$ and then decreases. Any tendency for the comparison-stimulus setting to be accommodated to the first overtone would thus be expected to be maximal for complex tones with fundamental frequencies of about $350 \mathrm{~Hz}$, which is quite consistent with the findings of Experiment 1.

Although this account within the context of Terhardt et al.'s model is highly speculative, it does suggest promising lines for future research. There is clearly a need for experimental identification of factors that determine the relative dominance of analytic and holistic modes in the perception of complex tones. It would also be worthwhile to attempt to obtain both sharpening and flattening of complex tones relative to simple tones in the same study, using subject selection and contextual, instructional, or training manipulations with face validity for the analytic-holistic distinction.

\section{REFERENCES}

BAnnister, H. (1934). Auditory phenomena and their stimulus correlations. In C. Murchison (Ed.), Handbook of general experimental psychology. Worcester, MA: Clark University Press.

Campbell, R. A., \& Small, A. M. (1963). Effect of practice and feedback on frequency discrimination. Journal of the Acoustical Society of America, 35, 1511-1514.

CARLSEN, J. C. (1981). Auditory perception: Concerns for musical learn- ing. In Documentary report of the Ann Arbor Symposium: Applications of psychology to the teaching and learning of music (pp. 2-8). Reston, VA: Music Educators National Conference.

Fletcher, H., \& Munson, W. A. (1933). Loudness, its definition, measurement and calculation. Journal of the Acoustical Society of America, 5, 82-108.

GoLDSTEIN, J. L. (1973). An optimum processor theory for the central formation of the pitch of complex tones. Journal of the Acoustical Society of America, 54, 1496-1516.

GreER, R. D. (1970). The effect of timbre on brass-wind intonation. In E. Gordon (Ed.), Experimental research in the psychology of music (pp. 65-94). Iowa City: University of Iowa Press.

HenNing, G. B., \& Grosberg, S. L. (1968). Effect of harmonic components on frequency discrimination. Journal of the Acoustical Society of America, 44, 1386-1389.

Lichte, W. H. (1941). Attributes of complex tones. Joumal of Experimental Psychology, 28, 455-465.

SEASHORE, C. E. (1938). Psychology of music. New York: MoGraw-Hill.

SERGEANT, D. (1973). Measurement of pitch perception. Journal of Research in Music Education, 21, 3-19.

SPIEGEL, M. F., \& Watson, C. S. (1984). Performance on frequencydiscrimination tasks by musicians and nonmusicians. Journal of the Acoustical Society of America, 76, 1690-1695.

TERHARDT, E. (1970). Frequency analysis and periodicity detection in the sensations of roughness and periodicity pitch. In R. Plomp (Ed.), Frequency analysis and periodicity detection in hearing (pp. 278-287). Leiden, The Netherlands: Sijthoff.

TERHARDT, E. (1971). Die Tonhohe harmonischer Klange und das Oktavintervall. Acustica, 24, 126-136.

TERHARdt, E., Stoll, G., \& SEewann, M. (1982). Algorithm for extraction of pitch and pitch salience from complex tonal signals. Journal of the Acoustical Society of America, 71, 679-688.

WARD, W. D. (1970). Musical perception. In J. V. Tobias (Ed.), Foundations of modern auditory theory (Vol. 1, pp. 405-447). New York: Academic Press.

Wier, C. C., Jesteadt, W., \& Green, D. M. (1976). A comparison of method-of-adjustment and forced-choice procedures in frequency discrimination. Perception \& Psychophysics, 19, 75-79.

Wightman, F. L. (1973). The pattern-transformation model of pitch. Journal of the Acoustical Society of America, 54, 407-416.

WyatT, R. F. (1945). Improvability of pitch discrimination. Psychological Monographs, 58, 1-58.

ZEITLIN, L. R. (1964). Frequency discrimination of pure and complex tones. Journal of the Acoustical Society of America, 36, 1027.

\section{NOTE}

1. Range appended to summary statistics in this paper represent \pm 1 standard error of the statistic.

(Manuscript received August 21, 1984; revision accepted for publication October 31,1985 .) 